\title{
ТРАЕКТОРНОЕ УПРАВЛЕНИЕ НАБЛЮДЕНИЯМИ В 3D С БОРТА БПЛА ПО УГЛОМЕРНОЙ ИНФОРМАЦИИ ${ }^{1}$
}

\author{
Д. Д. Емельянов, Е. Я. Рубинович
}

\begin{abstract}
B 3D рассматривается задача управления траекторией беспилотного летательного аппарата (БПЛА). Цель управления - повышение точности оценивания пространственных координат БПЛА по дискретным угловым измерениям положения некоторого неподвижного маяка в процессе наведения БПЛА на заданное терминальное множество. Измерения зашумлены дискретным белым шумом с известными характеристиками. Общее время наведения задано.

Ключевые слова: БПЛА, угловые измерения, управление наблюдениями, терминальное наведение.

D. D. Emeliyanov, E. Ya. Rubinovich. Trajectory control of 3D observations from an UAV by angular measurements.

We consider the 3D problem of controlling the trajectory of an unmanned aerial vehicle (UAV). The purpose of the control is to increase the accuracy of estimating the spatial coordinates of the UAV from discrete angular measurements of the position of a fixed beacon in the process of guidance of the UAV to a given terminal set. The measurements are contaminated by a discrete white noise with known characteristics. The total guidance time is specified.
\end{abstract}

Keywords: UAV, angular measurements, control of observations, terminal guidance.

MSC: 49N30, 49N35, 93B52

DOI: $10.21538 / 0134-4889-2020-26-1-112-130$

\section{Введение}

При выполнении автономных миссий беспилотными летательными аппаратами (БПЛА) часто ставится навигационная задача определения на борту БПЛА собственных элементов движения (координат и скоростей) на основе текущей информации от неподвижного радиомаяка. Однако точность оценки этих элементов движения (ЭД) существенно зависит от траектории, по которой БПЛА, играя роль мобильного наблюдателя, движется в процессе наблюдения (слежения), что связано с проблемой наблюдаемости [1].

В связи с этим естественно возникает проблема построения в реальном масштабе времени рациональной (с точки зрения повышения точности оценки ЭД) траектории движения БПЛА. Другими словами - задача траекторного управления наблюдениями с борта подвижного наблюдателя.

Целью данной работы является оптимизация описанного выше процесса слежения.

Фактически процесс слежения состоит из двух взаимосвязанных процессов: процесса сбора информации о цели (объекте наблюдения) и процесса обработки этой информации. Управление процессом сбора информации называется управлением наблюдениями, а сам процесс обработки наблюдений называется фильтрацией.

Сложность построения оптимальных процедур сбора и обработки информации при движении в реальном времени напрямую связана с нелинейностью как уравнений движения системы "наблюдатель-цель", так и уравнений, описывающих угловые измерения (пеленг цели и угол ее возвышения) $[2 ; 3]$.

Для получения оценок ЭД могут быть использованы хорошо известные расширенные (линеаризованные в окрестности текущей оценки) фильтры Калмана (EKF фильтры), методы

\footnotetext{
${ }^{1}$ Работа выполнена при частичной поддержке Программы 7 Президиума РАН.
} 
псевдоизмерений [4] или различные байесовские методы фильтрации: метод Монте-Карло [5] или метод гауссовской суммы [6]. Последние методы могут быть легко распространены на случай нескольких датчиков [7]. Однако в [8] показано, что оценки ЭД, полученные с помощью приведенных выше методов фильтрации, имеют примерно одинаковую точность.

Из-за габаритных и энергетических ограничений бортовые вычислители БПЛА, как правило, являются относительно малопроизводительными, что требует достаточно простых (рекуррентных) алгоритмов фильтрации, построенных, например, на основе расширенного (линеаризованного) фильтра Калмана. "Простота" таких алгоритмов и, в частности, ошибки линеаризации компенсируются лучшей информацией о цели, получаемой при оптимизации процесса наблюдения. Численные эксперименты показывают, что линеаризованный фильтр Калмана с траекторным управлением наблюдениями дает оценки ЭД не хуже, а иногда и лучше, чем сложные нелинейные алгоритмы фильтрации при произвольном (не оптимальном) движении наблюдателя [9].

В случае локализации неподвижной цели одна задача построения оптимальной траектории БПЛА была исследована в [2].

Один субоптимальный метод построения траекторий БПЛА рассмотрен в [3], где управление БПЛА на фиксированном временном интервале $[0, T]$ строится в виде линейной комбинации первых $\mathrm{N}$ базисных функций некоторого ортонормированного на $[0, T]$ базиса. В качестве такого базиса предлагается система ортогональных многочленов Чебышева.

Такой подход позволяет свести задачу оптимального управления к задаче нахождения максимума функций многих переменных (коэффициентов разложения по базису). Однако выбор числа $\mathrm{N}$ в [3] не обсуждается, поэтому получить оценки потерь в значении функционала при переходе к субоптимальному методу решения задачи не представляется возможным.

В [10] задача оптимального управления БПЛА ставится в терминах частично наблюдаемых марковских процессов принятия решений. В частности, рассматривается задача построения траектории БПЛА, осуществляющего наблюдения по азимуту и углу места в течение фиксированного интервала наблюдения $[0, T]$ за движущейся целью. Соответствующие задачи оптимального управления Майера сформулированы и решены численно с использованием принципа максимума Л.С. Понтрягина.

В настоящей статье рассматривается пространственная задача управления движущимся наблюдателем, который оценивает вектор своих координат и строит свое управление на основе дискретных измерений углового положения неподвижного маяка, расположенного в начале координат.

Специфика задачи заключается в том, что наблюдатель (БПЛА) имеет оптическую систему визирования с фиксированным фокусным расстоянием, которая в силу своих конструктивных особенностей способна измерять тангенсы углов азимута и возвышения (а не сами углы!) с некоторыми ошибками, которые мы будем считать независимыми и нормально распределенными. Требуется построить управление наблюдателем, которое переводило бы его за заданное время на некоторое конечное многообразие и одновременно повышало бы точность оценки трехмерного координатного вектора наблюдателя с помощью EKF-фильтра.

При решении этой задачи используется подход, изложенный в [11], который обобщается на случай пространственного движения наблюдателя. Близкая постановка рассматривалась в [9] с иной динамикой наблюдателя, где измерению были доступны собственно угловые координаты цели, а не их тангенсы.

\section{1. Постановка задачи}

\section{1. Геометрия задачи}

Рассмотрим пространственную задачу управления точечным объектом - наблюдателем $\mathrm{P}$ - по угломерной информации. Для описания движения объекта $\mathrm{P}$ введем декартову систему координат OXYZ (рис. 1). 


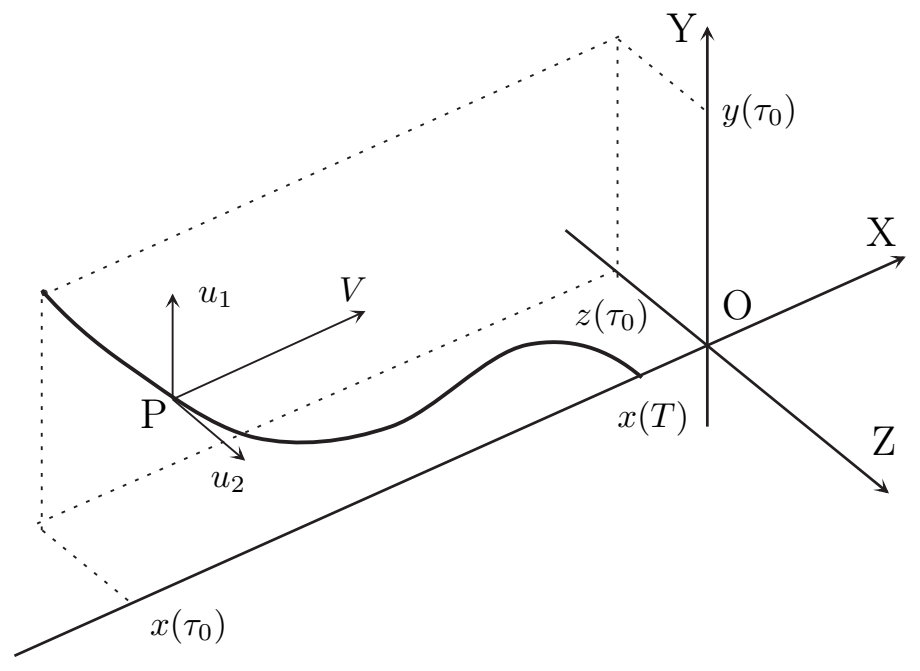

Рис. 1. К постановке задачи

$\mathrm{B}$ начальный момент времени $t=\tau_{0}$ наблюдатель $\mathrm{P}$ находится в точке с координатами $\left(x\left(\tau_{0}\right), y\left(\tau_{0}\right), z\left(\tau_{0}\right)\right)$. Дальнейшее его движение описывается следующей системой уравнений:

$$
\dot{x}=V, \quad \dot{y}=u_{1}, \quad \dot{z}=u_{2} .
$$

Здесь

$x=x(t), y=y(t), z=z(t)$ - компоненты радиус-вектора $R(t)$ наблюдателя в системе OXYZ;

$u=\left(u_{1}(t), u_{2}(t)\right)$ - двумерный вектор управления наблюдателя;

$V$ - заданная постоянная скорость, $V>0$.

Управляя скоростями $u_{1}(t)$ и $u_{2}(t)$ своего движения вдоль осей OY и OZ, наблюдатель должен в заданный момент $t=T$ попасть на ось OX. Особенностью динамики наблюдателя является то, что его движение вдоль оси $\mathrm{OX}$ (со скоростью $V$ ) не зависит от управления.

Предполагается, что точные координаты начальной точки $\left(x\left(\tau_{0}\right), y\left(\tau_{0}\right), z\left(\tau_{0}\right)\right)$ не известны, задана только их априорная оценка

$$
Z=\left(Z_{x}, Z_{y}, Z_{z}\right)
$$

и известно, что $x\left(\tau_{0}\right)<V\left(\tau_{0}-T\right)$, т. е. наблюдатель Р всегда находится слева от плоскости OYZ.

В процессе движения наблюдатель производит в дискретные моменты времени $t_{i}=\tau_{0}+i \Delta$ измерения $\zeta_{i}=\zeta\left(t_{i}\right)$ вида (тангенсы углов возвышения и азимута относительно неподвижного маяка, расположенного в точке О начала координат)

$$
\zeta_{1 i}=\frac{y_{i}}{x_{i}}+\sigma_{0} \eta_{1 i}, \quad \zeta_{2 i}=\frac{z_{i}}{x_{i}}+\sigma_{0} \eta_{2 i}, \quad i=1,2, \ldots
$$

Здесь

$\zeta_{i}=\left(\zeta_{1 i}, \zeta_{2 i}\right)-$ двумерный вектор измерений;

$x_{i}=x\left(t_{i}\right), y_{i}=y\left(t_{i}\right), z_{i}=z\left(t_{i}\right), \eta_{i}=\left(\eta_{1 i}, \eta_{2 i}\right)$ - последовательность независимых гауссовских случайных векторов с нулевым средним и единичной ковариационной матрицей;

$\sigma_{0}$ - среднеквадратическая ошибка измерений;

$\Delta-$ интервал между измерениями, $\Delta \ll T-\tau_{0}$.

Задачей наблюдателя является построение оценки $\widehat{R}$ вектора $R=(x, y, z)$ к моменту $T$ по данным наблюдений $\zeta_{i}$. Для построения оценки $\widehat{R}=(\widehat{x}, \widehat{y}, \widehat{z})$ наблюдатель применяет расширенный фильтр Калмана (EKF-фильтр), алгоритм которого в рассматриваемом случае записывается следующим образом. 


\section{2. ЕКF-фильтр}

1) Одношаговая экстраполяция $(i=1,2, \ldots)$ :

$$
\begin{aligned}
& \tilde{x}_{i}=\widehat{x}_{i-1}+V \Delta, \quad \tilde{y}_{i}=\widehat{y}_{i-1}+\int_{t_{i-1}}^{t_{i}} u_{1}(s) d s, \\
& \tilde{z}_{i}=\widehat{z}_{i-1}+\int_{t_{i-1}}^{t_{i}} u_{2}(s) d s, \quad \tilde{\gamma}\left(t_{i}\right)=\gamma\left(t_{i-1}\right),
\end{aligned}
$$

где $\gamma(\cdot)$ - ковариационная матрица ошибок оценивания размера $3 \times 3$. Начальная оценка $\widehat{R}\left(t_{0}\right)$ и начальная ковариационная матрица $\gamma\left(t_{0}\right)$ фильтра Калмана имеют вид

$$
\begin{aligned}
& \widehat{x}\left(t_{0}\right)=Z_{x}, \quad \widehat{y}\left(t_{0}\right)=Z_{y}, \\
& \widehat{z}\left(t_{0}\right)=Z_{z}, \quad \gamma\left(t_{0}\right)=G,
\end{aligned}
$$

где $Z=\left(Z_{x}, Z_{y}, Z_{z}\right)$ - вектор априорной оценки, $G$ - заданная ковариационная матрица ошибок априорного оценивания.

2) Матрица коэффициента усиления:

$$
K_{i}=\tilde{\gamma}\left(t_{i}\right) H_{i}^{T}\left(H_{i} \tilde{\gamma}\left(t_{i}\right) H_{i}^{T}+\sigma_{0}^{2} I_{2}\right)^{-1}
$$

Здесь символ $T$ обозначает транспонирование, $I_{k}-$ единичная матрица размера $k \times k, H_{i}=$ $H\left(\widetilde{R}_{i}\right)$ - линеаризованная в окрестности прогнозируемой одношаговой оценки матрица наблюдений вида

$$
H(\widetilde{R})=\frac{1}{\widetilde{x}}\left[\begin{array}{ccc}
-\varphi(\widetilde{R}) & 1 & 0 \\
-\psi(\widetilde{R}) & 0 & 1
\end{array}\right],
$$

где введены обозначения: $\widetilde{R}=(\widetilde{x}, \widetilde{y}, \widetilde{z})$,

$$
\varphi(\widetilde{R})=\frac{\widetilde{y}}{\widetilde{x}}, \quad \psi(\widetilde{R})=\frac{\widetilde{z}}{\widetilde{x}} .
$$

3) Оценка фильтрации и ковариационная матрица ошибок оценивания:

$$
\widehat{R}\left(t_{i}\right)=\widetilde{R}\left(t_{i}\right)+K_{i}\left[\begin{array}{c}
\zeta_{1 i}-\varphi\left(\widetilde{R}\left(t_{i}\right)\right) \\
\zeta_{2 i}-\psi\left(\widetilde{R}\left(t_{i}\right)\right)
\end{array}\right], \quad \gamma\left(t_{i}\right)=\left(I_{3}-K_{i} H_{i}\right) \tilde{\gamma}\left(t_{i}\right)
$$

Требуется построить управление $u$, которое переводит наблюдателя $\mathrm{P}$ в момент $T$ на прямую ОХ по траектории, обеспечивающей эффективную работу применяемого фильтра Калмана. Критерием эффективности работы фильтра является точность оценивания компонент вектора $R$ к моменту $T$, которая характеризуется диагональными элементами ковариационной матрицы $\gamma(T)$. Поэтому в качестве критерия предлагается следующий функционал:

$$
J(T)=\sum_{j=1}^{3} \beta_{j} \gamma_{j j}(T),
$$

где $\beta_{j}, j=1,2,3,-$ весовые коэффициенты, определяющие чувствительность критерия $J$ к точности оценивания отдельных компонент вектора $R(T)$. 


\section{2. Вспомогательная задача оптимального управления}

Заметим, что поставленная задача является нелинейной задачей стохастической оптимизации с критерием (1.2), зависящим от ковариационной матрицы $\gamma(T)$. Эта задача содержит в себе следующую принципиальную трудность. Дело в том, что находясь в процессе движения в произвольной точке $(R(t), \gamma(t))$, вычислить будущее значение матрицы $\gamma(T)$ не представляется возможным. В самом деле, как видно из уравнений расширенного фильтра Калмана, элементы матрицы $\gamma(T)$ суть случайные величины, зависящие от всей реализации наблюдений $\left\{\zeta_{i}\right\}$ от момента $\tau_{0}$ до момента $T$. Данная зависимость обусловлена тем, что в выражение для линеаризованной матрицы наблюдений $H(\cdot)$ явно входят компоненты экстраполированного вектора $\widetilde{R}$, который, в свою очередь, зависит от вектора оценки $\widehat{R}$ и, следовательно, от реализации наблюдений. В текущий же момент $t$ реализация будущих наблюдений, очевидно, неизвестна.

Создавшиеся проблемы снимет переход к так называемым условно-программным управлениям наблюдателя. Суть такого подхода - в следующем. Примем текущий момент времени $\tau$ за начальный $t_{0}$, а текущую оценку вектора $\widehat{R}(\tau)$ - за точное его значение, т. е. оценка $\widehat{R}(\tau)$ принимается за точно известную начальную позицию наблюдателя. Тогда траектория наблюдателя $R(t)$ при $t>\tau$ находится путем интегрирования уравнений (1.1) с управлением $u(t)$. В случае частых наблюдений дальнейшую эволюцию ковариационной матрицы $\gamma(t)$ при $t>\tau$ можно аппроксимировать дифференциальным уравнением [12, теорема 10.3]

$$
\dot{\Gamma}(t)=-\frac{1}{\sigma^{2}} \Gamma(t) H^{T}(R(t)) H(R(t)) \Gamma(t),
$$

где $\Gamma(\tau)=\gamma(\tau), \sigma=\sigma_{0} \sqrt{\Delta}$. В рамках такой аппроксимации значение матрицы $\gamma(T)$ будем считать известным и равным значению $\Gamma(T)$, которое находится путем интегрирования уравнения $(2.1)$ с начальным условием $\gamma(\tau)$ вдоль известной траектории $R(t)$. Таким образом, уравнения (2.1) и (1.1) задают связь между терминальным значением матрицы $\gamma(T)$ и управлением наблюдателя.

Найдем оптимальное управление $u^{*}(t)$, переводящее наблюдателя из точки $R(\tau)$ на ось $\mathrm{OX}$ в момент $T$ и минимизирующее терминальный критерий как функцию аппроксимированной матрицы $\gamma(T)$.

Пусть эта вспомогательная задача оптимального управления решена и найдено оптимальное управление из класса программных, т. е являющихся функциями времени, начального момента $\tau$, начальной позиции $\widehat{R}(\tau)$ и начальной ковариационной матрицы $\gamma(\tau)$.

Далее в течение некоторого отрезка времени $\Delta \tau$ наблюдатель движется по выработанной программе, обрабатывая результаты наблюдений, после чего производится корректировка программного управления, т. е. вычисление нового оптимального управления как функции времени, нового начального момента, новой начальной позиции и обновленной ковариационной матрицы. Управление, получаемое описанным способом, и называется условно-программным.

Фактически в рамках данного подхода решение стохастической задачи траекторного управления наблюдениями сводится к решению вспомогательной задачи оптимального управления с периодически обновляющимися начальными условиями и терминальным условием, означающим попадание наблюдателя на ось ОХ в момент $T$. Критерий оптимизации при этом должен характеризовать качество оценивания компонент вектора $R$ к моменту $T$.

Применим описанный подход для решения рассматриваемой задачи. В качестве критерия оптимизации для вспомогательной задачи возьмем следующий функционал

$$
J(T)=\sum_{j=1}^{3} \beta_{j} \Gamma_{j j}(T)+\int_{t_{0}}^{T}\left(u_{1}^{2}(s)+u_{2}^{2}(s)\right) d s,
$$


где $\Gamma_{j j}, j=1,2,3$ - диагональные элементы матрицы $\Gamma, \beta_{j}$ - положительные весовые коэффициенты. Заметим, что критерий (2.2) отличается от критерия (1.2) дополнительно введенной интегральной частью, которая предназначена для предотвращения появления реализаций условно-программного управления, требующих значительного расхода управляющего воздействия, а также для регуляризации вспомогательной задачи при малых значениях весовых коэффициентов. Из критерия (2.2), в частности, видно, что матричная переменная $\Gamma(t)$, подчиняющаяся уравнению (2.1), играет роль дополнительной фазовой переменной в вспомогательной задаче оптимального управления.

Для дальнейших выкладок удобно в критерии $(2.2)$ перейти от матрицы $\Gamma(t)$ к обратной матрице $D(t)=\Gamma^{-1}(t)$. Эволюция матрицы $D(t)$ описывается уравнением

$$
\dot{D}(t)=\frac{1}{\sigma^{2}} H^{T} H \quad \text { с начальным условием } \quad D\left(t_{0}\right)=\gamma^{-1}\left(t_{0}\right) .
$$

Тогда математическая модель вспомогательной задачи оптимального управления наблюдателем Р принимает следующий вид.

1. Дифференциальные уравнения динамики управляемой системы при $t \in\left[t_{0}, T\right]$

$$
\begin{aligned}
& \dot{x}=V, \quad \dot{y}=u_{1}, \quad \dot{z}=u_{2} \quad \dot{r}=u_{1}^{2}+u_{2}^{2}, \\
& \dot{D}=\frac{1}{(\sigma x)^{2}}\left[\begin{array}{ccc}
\varphi^{2}+\psi^{2} & -\varphi & -\psi \\
-\varphi & 1 & 0 \\
-\psi & 0 & 1
\end{array}\right] \text {, }
\end{aligned}
$$

где $D=D(t), \varphi=\varphi(R(t)), \psi=\psi(R(t)), x=x(t), y=y(t), z=z(t) ; r=r(t)$ - характеризует расход управляющего воздействия к моменту $t$.

2. Начальные условия при $t=t_{0}$

$$
\begin{aligned}
& x\left(t_{0}\right)=x_{0}, \quad y\left(t_{0}\right)=y_{0}, \quad z\left(t_{0}\right)=z_{0}, \\
& r\left(t_{0}\right)=0, \quad D\left(t_{0}\right)=\gamma^{-1}\left(t_{0}\right) .
\end{aligned}
$$

3. Терминальные условия при $t=T$

$$
y(T)=0, \quad z(T)=0 .
$$

4. Критерий оптимизации

$$
J(T)=\sum_{j=1}^{3} \beta_{j}\left(D^{-1}(T)\right)_{j j}+r(T) \longrightarrow \min .
$$

При решении задачи (2.3)-(2.7) рассмотрим вначале случай произвольных весовых коэффициентов $\beta_{j}, j=1,2,3$, после чего на основании полученных результатов исследуем частный случай $\beta_{2}=0, \beta_{3}=0$, при котором, как следует из вида функционала $(2.7)$, нас интересует точность оценивания только $x$-компоненты вектора $R$ на момент $T$.

\section{3. Решение вспомогательной задачи оптимального управления}

Для решения задачи (2.3)-(2.7) применим необходимые условия оптимальности в форме принципа максимума. В соответствии с этой методикой запишем функцию Гамильтона для рассматриваемой задачи

$$
\begin{gathered}
\mathcal{H}=\lambda_{1} V+\lambda_{2} u_{1}+\lambda_{3} u_{2}+\lambda_{4}\left(u_{1}^{2}+u_{2}^{2}\right) \\
+\frac{1}{(\sigma x)^{2}}\left(\lambda_{5}\left(\varphi^{2}+\psi^{2}\right)-\left(\lambda_{6}+\lambda_{7}\right) \varphi-\left(\lambda_{8}+\lambda_{9}\right) \psi+\lambda_{10}+\lambda_{11}\right),
\end{gathered}
$$


где $\lambda=\left(\lambda_{1}, \ldots, \lambda_{11}\right)$ - вектор сопряженных переменных (множителей Лагранжа), не обращающихся в нуль одновременно. Здесь $\lambda_{1}, \ldots, \lambda_{4}$ отвечают переменным $(2.3)$, а $\lambda_{5}, \ldots, \lambda_{11}-$ переменным $d_{11}, d_{12}, d_{21}, d_{13}, d_{31}, d_{22}, d_{33}$ соответственно, где $d_{i j}, i, j=1,2,3$, - элементы матрицы $D(T)$. Сопряженные переменные удовлетворяют следующей системе уравнений:
а) $\dot{\lambda}_{1}=s(x)\left(4 \lambda_{5}\left(\varphi^{2}+\psi^{2}\right)-3\left(\lambda_{6}+\lambda_{7}\right) \varphi-3\left(\lambda_{8}+\lambda_{9}\right) \psi+2\left(\lambda_{10}+\lambda_{11}\right)\right)$,
б) $\dot{\lambda}_{2}=s(x)\left(\lambda_{6}+\lambda_{7}-2 \lambda_{5} \varphi\right)$,
в) $\dot{\lambda}_{3}=s(x)\left(\lambda_{8}+\lambda_{9}-2 \lambda_{5} \psi\right)$,
г) $\dot{\lambda}_{i}=0, \quad i=4, \ldots, 11$,

где $s(x)=\left(\sigma^{2} x^{3}\right)^{-1}$. Из уравнений $(3.1$ г) следует, что

$$
\lambda_{i}=\text { const }, \quad i=4, \ldots, 11 .
$$

В соответствии с принципом максимума в момент $T$ имеет место условие трансверсальности

$$
\begin{gathered}
\lambda_{1} \delta x+\lambda_{2} \delta y+\lambda_{3} \delta z+\left(\lambda_{4}+1\right) \delta r+\left(\lambda_{5}+\kappa_{11}\right) \delta d_{11} \\
+\left(\lambda_{6}+\kappa_{12}\right) \delta d_{12}+\left(\lambda_{7}+\kappa_{21}\right) \delta d_{21}+\left(\lambda_{8}+\kappa_{13}\right) \delta d_{13} \\
+\left(\lambda_{9}+\kappa_{31}\right) \delta d_{31}+\left(\lambda_{10}+\kappa_{22}\right) \delta d_{22}+\left(\lambda_{11}+\kappa_{33}\right) \delta d_{33}=0,
\end{gathered}
$$

где $\kappa_{i j}$ - частные производные функционала (2.7) по элементам $d_{i j}$ матрицы $D(T), i, j=1,2,3$.

Из (2.6) следует, что вариации $\delta y$ и $\delta z$ в момент $T$ равны нулю, а остальные вариации, в частности $\delta r$ и $\delta d_{i j}$, произвольны. С учетом этого обстоятельства и равенств $(3.2)$ находим

$$
\begin{array}{llll}
\lambda_{4} \equiv-1, & \lambda_{5} \equiv-\kappa_{11}, & \lambda_{6} \equiv-\kappa_{12}, & \lambda_{7} \equiv-\kappa_{21}, \\
\lambda_{8} \equiv-\kappa_{13}, & \lambda_{9} \equiv-\kappa_{31}, & \lambda_{10} \equiv-\kappa_{22}, & \lambda_{11} \equiv-\kappa_{33} .
\end{array}
$$

Дифференцирование функционала (2.7) по $d_{i j}$ с учетом симметричности матрицы $D(T)$ дает

$$
\begin{aligned}
& \kappa_{11}=-\sum_{j=1}^{3} \beta_{j}\left(D^{-1}(T)\right)_{1 j}^{2}, \\
& \kappa_{12} \equiv \kappa_{21}=-\beta_{3} \frac{d_{12}}{m}-\left(D^{-1}(T)\right)_{12} \sum_{j=1}^{3} \beta_{j}\left(D^{-1}(T)\right)_{j j}, \\
& \kappa_{13} \equiv \kappa_{31}=-\beta_{2} \frac{d_{13}}{m}-\left(D^{-1}(T)\right)_{13} \sum_{j=1}^{3} \beta_{j}\left(D^{-1}(T)\right)_{j j},
\end{aligned}
$$

где введено обозначение $m=\operatorname{det} D(T)$.

Оптимальное управление $u^{*}(t)=\left(u_{1}^{*}(t), u_{2}^{*}(t)\right)$ должно доставлять максимум гамильтониану $\mathcal{H}$. Так как ограничения на компоненты $u_{1}, u_{2}$ отсутствуют, условия максимума $\mathcal{H}$ дают

$$
\begin{aligned}
& \frac{\partial \mathcal{H}}{\partial u_{1}}=\lambda_{2}+2 \lambda_{4} u_{1}=0, \quad \frac{\partial^{2} \mathcal{H}}{\partial u_{1}^{2}}=2 \lambda_{4} \equiv-2<0, \\
& \frac{\partial \mathcal{H}}{\partial u_{2}}=\lambda_{3}+2 \lambda_{4} u_{2}=0, \quad \frac{\partial^{2} \mathcal{H}}{\partial u_{2}^{2}}=2 \lambda_{4} \equiv-2<0 .
\end{aligned}
$$

С учетом тождества $\lambda_{4} \equiv-1$ оптимальные управления принимают вид

$$
u_{1}^{*}(t)=\frac{\lambda_{2}(t)}{2}, \quad u_{2}^{*}(t)=\frac{\lambda_{3}(t)}{2} .
$$


Подставим оптимальные управления (3.4) в уравнения движения (2.3):

$$
\dot{y}(t)=\frac{\lambda_{2}(t)}{2}, \quad \dot{z}(t)=\frac{\lambda_{3}(t)}{2} .
$$

Продифференцируем по времени правые и левые части данных соотношений и подставим в правые части получившихся после дифференцирования уравнений выражения для $\dot{\lambda}_{2}$ и $\dot{\lambda}_{3}$ из $(3.1$ б,в). С учетом условий (3.3) выводим

$$
\begin{aligned}
& \frac{d^{2} y}{d t^{2}}=\frac{\dot{\lambda}_{2}}{2}=\frac{1}{\sigma^{2} x^{3}}\left(\kappa_{11} \frac{y}{x}-\kappa_{12}\right) \\
& \frac{d^{2} z}{d t^{2}}=\frac{\dot{\lambda}_{3}}{2}=\frac{1}{\sigma^{2} x^{3}}\left(\kappa_{11} \frac{z}{x}-\kappa_{13}\right)
\end{aligned}
$$

Сделаем в этих уравнениях замену аргумента и перейдем от времени $t$ к координате $x$ по формуле, вытекающей из уравнения движения наблюдателя вдоль оси ОХ:

$$
t=\frac{x-x_{0}}{V}, \quad \frac{d}{d x}=\frac{1}{V} \frac{d}{d t} .
$$

После замены аргумента получаем систему дифференциальных уравнений второго порядка, которая определяет вид оптимальной траектории $y(x), z(x)$. Имеем

$$
y^{\prime \prime}=-\varepsilon^{2} \frac{y}{x^{4}}+\varepsilon^{2} \frac{B_{1}}{x^{3}}, \quad z^{\prime \prime}=-\varepsilon^{2} \frac{z}{x^{4}}+\varepsilon^{2} \frac{B_{2}}{x^{3}},
$$

где ' обозначает дифференцирование по $x, x=x_{0}+V\left(t-t_{0}\right)$, а

$$
\varepsilon^{2}=-\frac{\kappa_{11}}{(\sigma V)^{2}}, \quad B_{1}=\frac{\kappa_{12}}{\kappa_{11}}, \quad B_{2}=\frac{\kappa_{13}}{\kappa_{11}} .
$$

Общее решение системы (3.5) выглядит следующим образом:

$$
\begin{aligned}
& y(x)=A_{1} x \sin \left(\frac{\varepsilon}{x}+f_{1}\right)+B_{1} x, \\
& z(x)=A_{2} x \sin \left(\frac{\varepsilon}{x}+f_{2}\right)+B_{2} x
\end{aligned}
$$

где $A_{i}, f_{i}, i=1,2,-$ константы, которые находятся из краевых условий $(2.5),(2.6)$ для функций $x(t), y(t), z(t)$. Эти условия дают

$$
\begin{aligned}
& A_{1} \sin \left(\frac{\varepsilon}{x_{0}}+f_{1}\right)=\varphi_{0}-B_{1}, \quad A_{1} \sin \left(\frac{\varepsilon}{a}+f_{1}\right)=-B_{1}, \\
& A_{2} \sin \left(\frac{\varepsilon}{x_{0}}+f_{2}\right)=\psi_{0}-B_{2}, \quad A_{2} \sin \left(\frac{\varepsilon}{a}+f_{2}\right)=-B_{2},
\end{aligned}
$$

где $\varphi_{0}=\varphi\left(R\left(t_{0}\right)\right), \psi_{0}=\psi\left(R\left(t_{0}\right)\right), a=x(T)=x_{0}+V\left(T-t_{0}\right)$. Решая данную систему уравнений относительно $A_{i}$ и $f_{i}, i=1,2$, и подставляя результат в $(3.7),(3.8)$, получаем

$$
\begin{aligned}
& y(x)=\frac{x}{\sin \xi}\left(B_{1} \sin \left(\frac{\varepsilon}{x}-\frac{\varepsilon}{x_{0}}\right)+\left(\varphi_{0}-B_{1}\right) \sin \left(\frac{\varepsilon}{x}-\frac{\varepsilon}{a}\right)\right)+B_{1} x, \\
& z(x)=\frac{x}{\sin \xi}\left(B_{2} \sin \left(\frac{\varepsilon}{x}-\frac{\varepsilon}{x_{0}}\right)+\left(\psi_{0}-B_{2}\right) \sin \left(\frac{\varepsilon}{x}-\frac{\varepsilon}{a}\right)\right)+B_{2} x,
\end{aligned}
$$

где $\xi=\varepsilon\left(x_{0}^{-1}-a^{-1}\right)$. Заметим, что вид оптимальной траектории $(3.9),(3.10)$ не меняется при замене $\varepsilon$ на $-\varepsilon$, поэтому без ограничения общности можно считать, что $\varepsilon>0$. Характерный вид траекторий, описываемых уравнениями (3.9), (3.10) показан на рис. 2. 


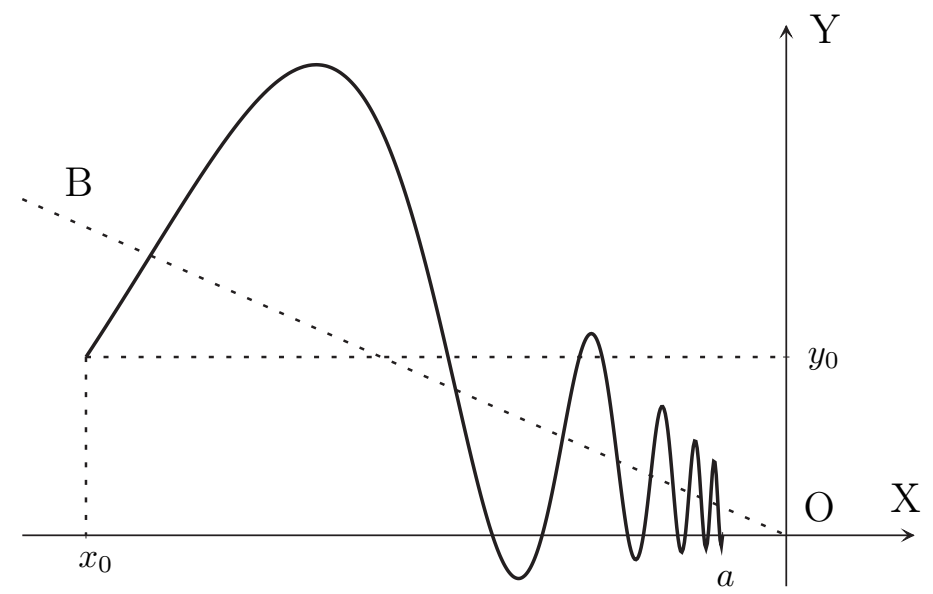

Рис. 2. Характерный вид траектории наблюдателя

Прямая ОВ, показанная пунктиром, имеет угловой коэффициент равный $B_{1}$. Из рис. 2 видно, что на траектории (3.9), (3.10) движение наблюдателя представляет собой характерное колебание относительно прямой $\mathrm{OB}$, амплитуда которого убывает с уменьшением модуля $x(t)$, а частота - возрастает. При этом параметр $\varepsilon$ имеет смысл начальной пространственной частоты колебаний.

Входящие в уравнения (3.9), (3.10) параметры $\varepsilon, B_{1}$ и $B_{2}$ задаются равенствами (3.6), которые после подстановки значений производных $\kappa_{11}, \kappa_{12}, \kappa_{13}$ принимают следующий вид

$$
\begin{aligned}
\varepsilon & =\frac{\sqrt{\beta_{1} \Gamma_{11}^{2}(T)+\beta_{2} \Gamma_{12}^{2}(T)+\beta_{3} \Gamma_{13}^{2}(T)}}{\sigma V} \\
B_{1} & =\frac{\Gamma_{12}(T)\left(\beta_{1} \Gamma_{11}(T)+\beta_{2} \Gamma_{22}(T)\right)+\beta_{3} \Gamma_{13}(T) \Gamma_{23}(T)}{\beta_{1} \Gamma_{11}^{2}(T)+\beta_{2} \Gamma_{12}^{2}(T)+\beta_{3} \Gamma_{13}^{2}(T)}, \\
B_{2} & =\frac{\Gamma_{13}(T)\left(\beta_{1} \Gamma_{11}(T)+\beta_{3} \Gamma_{33}(T)\right)+\beta_{2} \Gamma_{12}(T) \Gamma_{23}(T)}{\beta_{1} \Gamma_{11}^{2}(T)+\beta_{2} \Gamma_{12}^{2}(T)+\beta_{3} \Gamma_{13}^{2}(T)},
\end{aligned}
$$

где для краткости записи вместо элементов матрицы $D(T)$ подставлены соответствующие элементы матрицы $\Gamma(T)=D^{-1}(T)$. Сама матрица $D(T)$ находится путем интегрирования уравнений (2.4) на оптимальной траектории, описываемой уравнениями (3.9), (3.10). Результаты интегрирования имеют вид

$$
\begin{aligned}
d_{11}= & d_{11}\left(t_{0}\right)+L\left(2 Q(\xi)\left[B_{1}\left(\varphi_{0}-2 B_{1}\right)+B_{2}\left(\psi_{0}-2 B_{2}\right)\right]\right. \\
& +4 C(\xi)\left[B_{1}\left(\varphi_{0}-B_{1}\right)+B_{2}\left(\psi_{0}-B_{2}\right)\right] \\
& \left.+S(\xi)\left[\left(\varphi_{0}-2 B_{1}\right)^{2}+\left(\psi_{0}-2 B_{2}\right)^{2}\right]+B_{1}^{2}+B_{2}^{2}\right), \\
d_{12}= & d_{12}\left(t_{0}\right)-L\left(B_{1}+Q(\xi)\left(\varphi_{0}-2 B_{1}\right)\right), \\
d_{13}= & d_{13}\left(t_{0}\right)-L\left(B_{2}+Q(\xi)\left(\psi_{0}-2 B_{2}\right)\right), \\
d_{22}= & d_{22}\left(t_{0}\right)+L, \quad d_{23}=d_{23}\left(t_{0}\right), \\
d_{33}= & d_{33}\left(t_{0}\right)+L,
\end{aligned}
$$

где введены обозначения

$$
\begin{aligned}
Q(\xi) & =\frac{1}{\xi} \operatorname{tg} \frac{\xi}{2}, \quad L=\frac{1}{V \sigma^{2}}\left(\frac{1}{x_{0}}-\frac{1}{a}\right) \\
C(\xi) & =\frac{(1+\cos \xi)(\xi-\sin \xi)}{2 \xi(1-\cos 2 \xi)}, \quad S(\xi)=\frac{2 \xi-\sin 2 \xi}{2 \xi(1-\cos 2 \xi)} .
\end{aligned}
$$

Таким образом, искомое решение вспомогательной задачи оптимального управления (2.3)-(2.7) задается уравнениями (3.9), (3.10) с константами $\varepsilon, B_{1}$ и $B_{2}$, которые находятся из решения системы уравнений (3.11)-(3.14). 
Перейдем теперь к исследованию частного случая задачи (2.3)-(2.7), когда в критерии $(2.7)$ весовой коэффициент $\beta_{1}$ отличен от нуля, а коэффициенты $\beta_{2}, \beta_{3}$ равны нулю.

\section{1. Исследование частного случая вспомогательной задачи}

Как отмечалось в разделе, посвященном постановке вспомогательной задачи оптимального управления, мы полагаем $\beta_{2}=0, \beta_{3}=0$ в случае, когда требуется построить управление, повышающее точность оценивания $x$-компоненты вектора $R$ на момент $T$.

При $\beta_{2}=0, \beta_{3}=0$ уравнения (3.11)-(3.13) упрощаются и приводятся к следующему виду

$$
\begin{aligned}
& \text { а) } B_{1} d_{22}+B_{2} d_{23}+d_{12}=0, \\
& \text { б) } B_{1} d_{23}+B_{2} d_{33}+d_{13}=0, \\
& \text { в) } \sigma V \varepsilon\left(d_{11}-B_{1}^{2} d_{22}-2 B_{1} B_{2} d_{23}-B_{2}^{2} d_{33}\right)=\sqrt{\beta_{1}} .
\end{aligned}
$$

Подставляя в $\left(3.15\right.$ а,б) значения $d_{12}, d_{13}, d_{22}, d_{23}$ и $d_{33}$ из равенств $(3.14)$ и разрешая получившуюся систему уравнений относительно $B_{1}$ и $B_{2}$, находим

$$
\begin{aligned}
& B_{1}(\xi)=\frac{M_{12}+\mu(\xi)\left(\varphi_{0} d_{33}^{0}-\psi_{0} d_{23}^{0}-2 d_{12}^{0}\right)+2 \mu^{2}(\xi) \varphi_{0}}{M_{11}+2 \mu(\xi)\left(d_{22}^{0}+d_{33}^{0}\right)+4 \mu^{2}(\xi)}, \\
& B_{2}(\xi)=\frac{M_{13}+\mu(\xi)\left(\psi_{0} d_{22}^{0}-\varphi_{0} d_{23}^{0}-2 d_{13}^{0}\right)+2 \mu^{2}(\xi) \psi_{0}}{M_{11}+2 \mu(\xi)\left(d_{22}^{0}+d_{33}^{0}\right)+4 \mu^{2}(\xi)},
\end{aligned}
$$

где введены обозначения $d_{i j}^{0}=d_{i j}\left(t_{0}\right), \mu(\xi)=L Q(\xi)$ и

$$
\begin{aligned}
& M_{12}=d_{23}^{0} d_{13}^{0}-d_{12}^{0} d_{33}^{0}, \quad M_{13}=d_{23}^{0} d_{12}^{0}-d_{13}^{0} d_{22}^{0} \\
& M_{11}=d_{22}^{0} d_{33}^{0}-\left(d_{23}^{0}\right)^{2} .
\end{aligned}
$$

Заметим, что выражения для $B_{1}(\xi), B_{2}(\xi)$ можно представить в следующем виде:

$$
B_{1}(\xi)=\frac{\varphi_{0}}{2}-W_{1}(\xi), \quad B_{2}(\xi)=\frac{\psi_{0}}{2}-W_{2}(\xi)
$$

Здесь

$$
\begin{aligned}
& W_{1}(\xi)=\frac{\varphi_{0} M_{11} / 2-M_{12}+\mu(\xi)\left(\varphi_{0} d_{22}^{0}+\psi_{0} d_{23}^{0}+2 d_{12}^{0}\right)}{M_{11}+2 \mu(\xi)\left(d_{22}^{0}+d_{33}^{0}\right)+4 \mu^{2}(\xi)}, \\
& W_{2}(\xi)=\frac{\psi_{0} M_{11} / 2-M_{13}+\mu(\xi)\left(\psi_{0} d_{33}^{0}+\varphi_{0} d_{23}^{0}+2 d_{13}^{0}\right)}{M_{11}+2 \mu(\xi)\left(d_{22}^{0}+d_{33}^{0}\right)+4 \mu^{2}(\xi)} .
\end{aligned}
$$

После подстановки в $\left(3.15\right.$ в) значений $d_{11}, d_{22}, d_{23}, d_{33}$ и равенств $(3.16)$, уравнение (3.15 в) приводится к виду

$$
\begin{aligned}
\frac{\sigma L \sqrt{\beta_{1}}}{\xi}= & d_{11}^{0}+B_{1}^{2}(\xi) d_{22}^{0}+B_{2}^{2}(\xi) d_{33}^{0}+2 B_{1}(\xi) d_{12}^{0}+2 B_{2}(\xi) d_{13}^{0}+2 B_{1}(\xi) B_{2}(\xi) d_{23}^{0} \\
& +\frac{L}{\xi}\left\{\left(\frac{\varphi_{0}^{2}}{4}+\frac{\psi_{0}^{2}}{4}\right) \frac{\xi-\sin \xi}{1-\cos \xi}+\left(W_{1}^{2}(\xi)+W_{2}^{2}(\xi)\right) \frac{\xi+\sin \xi}{1+\cos \xi}\right\}
\end{aligned}
$$

где $\xi$ можно рассматривать как новое неизвестное, введенное вместо $\varepsilon$; при этом $\xi>0$.

Предложение 1. Уравнение (3.17) всегда имеет решение. 
Д о к а з а т е л ь с т в о. Обозначим через $F_{1}(\xi)$ левую часть уравнения (3.17), а через $F_{2}(\xi)$ - правую часть этого уравнения и исследуем поведение функций $F_{1}(\xi)$ и $F_{2}(\xi)$ при $\xi \geq 0$.

Графики функций $F_{1}(\xi)$ и $F_{2}(\xi)$ показаны на рис. 3.

Отметим основные свойства функции $F_{2}(\xi)$. Во-первых, функция $F_{2}(\xi)$ положительна при $\xi>0$. Действительно, первые шесть слагаемых в правой части уравнения (3.17) можно рассматривать как функцию двух переменных $-B_{1}$ и $B_{2}$. Эта функция имеет вид

$$
\Phi\left(B_{1}, B_{2}\right)=d_{11}^{0}+2 B_{1} d_{12}^{0}+2 B_{2} d_{13}^{0}+B_{1}^{2} d_{22}^{0}+2 B_{1} B_{2} d_{23}^{0}+B_{2}^{2} d_{33}^{0} .
$$

В силу непрерывности функции $\Phi\left(B_{1}, B_{2}\right)$ по обоим аргументам, для доказательства того что $\Phi\left(B_{1}, B_{2}\right)>0$, достаточно показать, что $\min \Phi\left(B_{1}, B_{2}\right)>0$. Матрица $q$ вторых производных функции $\Phi$ имеет вид

$$
q=2\left[\begin{array}{ll}
d_{22}^{0} & d_{23}^{0} \\
d_{23}^{0} & d_{33}^{0}
\end{array}\right] .
$$

В силу положительной определенности матрицы $D\left(t_{0}\right)$ матрица $q$ также положительно определена. Следовательно экстремум функции $\Phi\left(B_{1}, B_{2}\right)$ является минимумом. Дифференцируя $\Phi\left(B_{1}, B_{2}\right)$ по $B_{1}$ и $B_{2}$, находим, что точка минимума $\left(B_{1}^{*}, B_{2}^{*}\right)$ определяется уравнениями

$$
d_{12}^{0}+B_{1} d_{22}^{0}+B_{2} d_{23}^{0}=0, \quad d_{13}^{0}+B_{2} d_{33}^{0}+B_{1} d_{23}^{0}=0 .
$$

Отсюда

$$
B_{1}^{*}=\frac{d_{23}^{0} d_{13}^{0}-d_{12}^{0} d_{33}^{0}}{d_{22}^{0} d_{33}^{0}-\left(d_{23}^{0}\right)^{2}}, \quad B_{2}^{*}=\frac{d_{23}^{0} d_{12}^{0}-d_{13}^{0} d_{22}^{0}}{d_{22}^{0} d_{33}^{0}-\left(d_{23}^{0}\right)^{2}}
$$

Подставляя эти значения в (3.18), вычисляем минимум функции $\Phi\left(B_{1}, B_{2}\right)$

$$
\Phi\left(B_{1}^{*}, B_{2}^{*}\right)=\frac{\operatorname{det} D\left(t_{0}\right)}{d_{22}^{0} d_{33}^{0}-\left(d_{23}^{0}\right)^{2}}=\frac{1}{\gamma_{11}\left(t_{0}\right)}>0 .
$$

Таким образом, функция $\Phi\left(B_{1}, B_{2}\right)$ положительна при всех значениях параметров $B_{1}, B_{2}$. Далее, выражение в фигурных скобках в правой части уравнения (3.17) также положительно, поскольку $\xi>|\sin \xi|$ при $\xi>0$; следовательно функция $F_{2}(\xi)$ положительна при всех $\xi>0$.

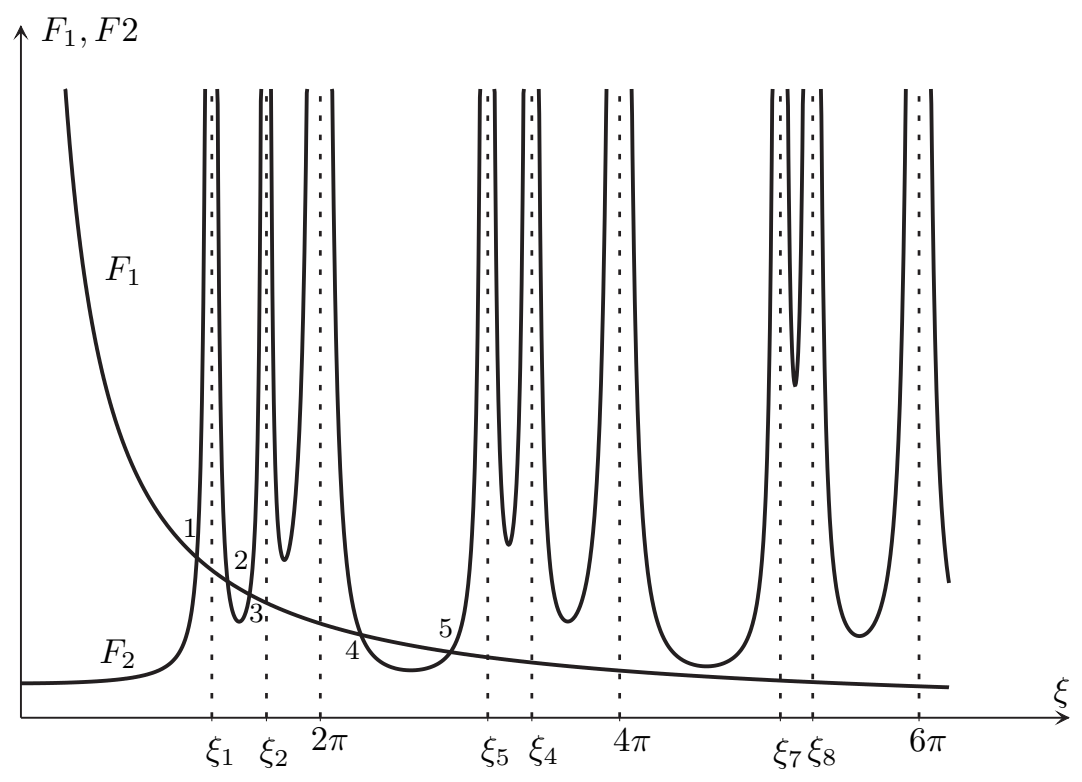

Рис. 3. Функции $F_{1}(\xi)$ и $F_{2}(\xi)$ 
Во-вторых, функция $F_{2}(\xi)$ имеет конечный положительный предел при $\xi \rightarrow+0$, так как существуют следующие пределы:

$$
\begin{aligned}
& \lim _{\xi \rightarrow+0}\left(\frac{\xi+\sin \xi}{\xi(1+\cos \xi)}\right)=1, \quad \lim _{\xi \rightarrow+0} \mu(\xi)=\frac{L}{2}, \\
& \lim _{\xi \rightarrow+0}\left(\frac{\xi-\sin \xi}{\xi(1-\cos \xi)}\right)=\frac{2}{3} .
\end{aligned}
$$

Кроме того, функция $F_{2}(\xi)$ стремится к $+\infty$ при $\xi \rightarrow \xi_{i}, i=1,2, \ldots$, где

$$
\xi_{i}=\left\{\begin{array}{l}
2 \pi n, \quad n=1,2, \ldots, \\
\text { корни уравнения } \\
M_{11}+2 \mu(\xi)\left(d_{22}^{0}+d_{33}^{0}\right)+4 \mu^{2}(\xi)=0,
\end{array}\right.
$$

Как показано на рис. 3 , график функции $F_{2}(\xi)$ имеет в точках $\xi_{i}, i=1,2, \ldots$, вертикальные асимптоты, отмеченные пунктиром.

В свою очередь функция $F_{1}(\xi)$, будучи гиперболой, непрерывна и положительна при $\xi>0$, монотонно стремится к нулю при $\xi \rightarrow+\infty$ и стремится к $+\infty$ при $\xi \rightarrow+0$ (рис. 3 ).

Описав поведение функций $F_{1}(\xi), F_{2}(\xi)$, перейдем к доказательству того, что уравнение (3.17) всегда имеет решение. Рассмотрим функцию $f(\xi)=F_{2}(\xi)-F_{1}(\xi)$ на интервале $\left(0, \xi_{1}\right)$. В силу свойств $F_{1}(\xi), F_{2}(\xi)$ функция $f(\xi)$ стремится к $-\infty$ при $\xi \rightarrow+0$ и к $+\infty$ при $\xi \rightarrow \xi_{1}$. Следовательно по определению бесконечного предела, на интервале $\left(0, \xi_{1}\right)$ всегда найдутся две точки $\omega_{1}, \omega_{2}$, такие что $f\left(\omega_{1}\right)<0$ и $f\left(\omega_{2}\right)>0$. Тогда в силу непрерывности функции $f(\xi)$ на отрезке $\left[\omega_{1}, \omega_{2}\right]$ есть хотя бы одна точка $\xi^{*}$, в которой $f\left(\xi^{*}\right)=0$, что эквивалентно существованию хотя бы одного решения уравнения (3.17).

Предложение доказано.

Таким образом, уравнение (3.17) всегда имеет решение, хотя, возможно, и не единственное. Например, на рис. 3 показан случай, когда существуют пять корней уравнения (3.17) (обозначены цифрами от 1 до 5). Неединственность решения означает, что необходимые условия оптимальности, из которых и было получено уравнение (3.17), не позволяют однозначно определить параметры $B_{1}(\xi), B_{2}(\xi)$ и $\varepsilon(\xi)$ и, следовательно, траекторию, доставляющие минимум функционалу (2.7) или эквивалентному ему функционалу (2.2). Чтобы устранить эту неоднозначность и выбрать один из корней уравнения (3.17), рассмотрим значение критерия (2.2) как функции $J^{*}(\xi)$ на траектории $(3.9),(3.10)$ при $\beta_{2}=0, \beta_{3}=0$. Имеем

$$
J^{*}(\xi)=J_{\Gamma}(\xi)+J_{R}(\xi),
$$

где через $J_{\Gamma}, J_{R}$ обозначены соответственно значения первого слагаемого (зависящего от $\Gamma(T)$ ) и второго слагаемого функционала $(2.2)$. График функции $J^{*}(\xi)$ показан на рис. 4 , где для наглядности представлены также графики функций $F_{1}(\xi), F_{2}(\xi)$ и обозначены точки их пересечения, т. е. решения уравнения (3.17).

Как следует из $(2.2)$, при $\beta_{2}=0, \beta_{3}=0$ величина $J_{\Gamma}(\xi)$ равна произведению весового коэффициента $\beta_{1}$ на значение элемента ковариационной матрицы $\Gamma_{11}(T)$, отвечающего за точность оценивания $x$-компоненты вектора $R(T)$. Непосредственные вычисления показывают, что на траектории (3.9), (3.10) при $\beta_{2}=0, \beta_{3}=0$ функция $J_{\Gamma}(\xi)$ имеет вид

$$
J_{\Gamma}(\xi)=\frac{\xi \sqrt{\beta_{1}}}{L} .
$$

Таким образом, на траектории (3.9), (3.10) величина $J_{\Gamma}(\xi)$ пропорциональна значению корня $\xi$ уравнения (3.17). Следовательно в рамках вспомогательной задачи оптимального управления максимальная точность оценивания величины $R_{x}(T)$ достигается при движении по траектории, соответствующей минимальному корню $\xi^{*}$ уравнения (3.17). 


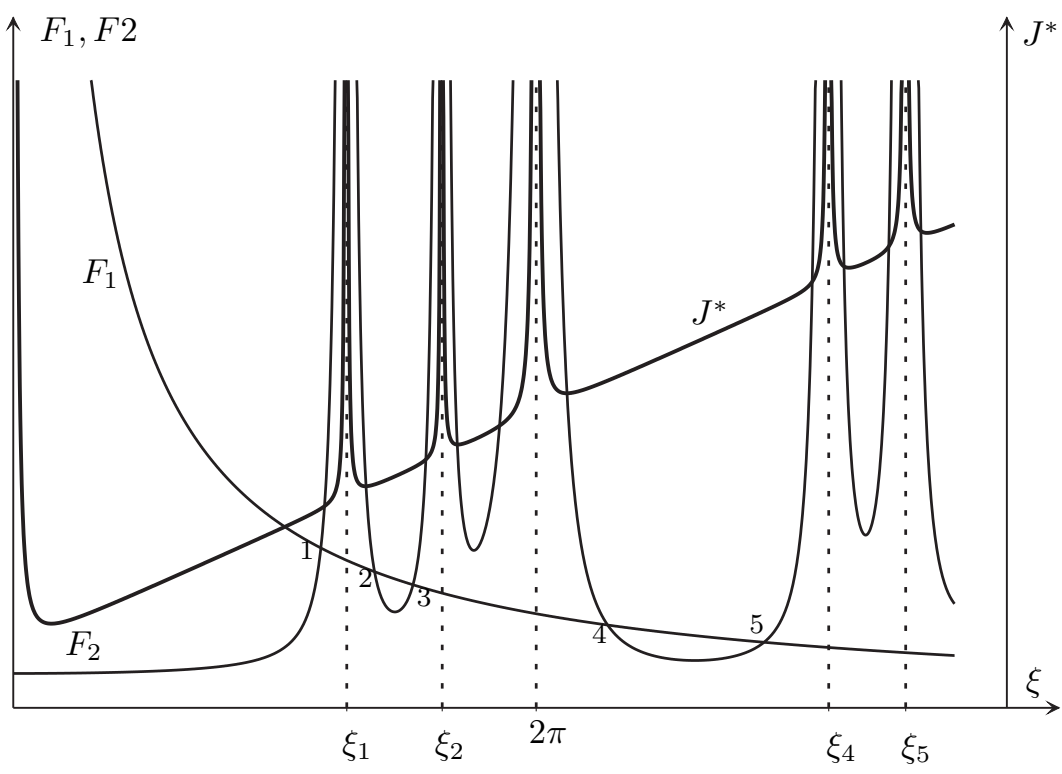

Рис. 4. Функция $J^{*}(\xi)$

Заметим, что слагаемое $J_{R}$ было добавлено в функционал (2.2) для регуляризации вспомогательной задачи оптимального управления при малых значениях весовых коэффициентов, а также для избежания значительного расхода управляющего воздействия на начальном участке условно-программной траектории при плохом знании компонент вектора $R(t)$. При этом главная цель управления наблюдателем состоит в повышении точности оценивания компонент вектора $R(T)$, т. е. в минимизации первого слагаемого функционала (2.2). С учетом этих замечаний выберем в качестве искомого значения $\xi$ минимальный корень $\xi^{*}$, который, как следует из (3.19), дает наименьшее по сравнению с остальными корнями уравнения (3.17) значение первой части $J_{\Gamma}$ функционала $(2.2)$.

Как было показано выше, минимальный корень $\xi^{*}$ уравнения (3.17) всегда существует и локализован на интервале $\left(0, \xi_{1}\right)$, где $\xi_{1}$ - минимальный корень уравнения

$$
M_{11}+2 \mu(\xi)\left(d_{22}^{0}+d_{33}^{0}\right)+4 \mu^{2}(\xi)=0 .
$$

Решая данное квадратное уравнение относительно $\mu$, получаем

$$
\mu_{1,2}=\frac{-d_{22}^{0}-d_{33}^{0} \pm \sqrt{\left(d_{22}^{0}-d_{33}^{0}\right)^{2}+4\left(d_{23}^{0}\right)^{2}}}{4},
$$

где знак "плюс" перед квадратным корнем соответствует решению $\mu_{1}$, а знак "минус" - peшению $\mu_{2}$. В силу положительной определенности матрицы $D\left(t_{0}\right)$ оба корня $-\mu_{1}$ и $\mu_{2}-$ отрицательны и $\mu_{2}<\mu_{1}$. Искомое значение $\xi_{1}$ будет минимальным ненулевым корнем уравнений

$$
\mu(\xi)=\mu_{1}, \quad \mu(\xi)=\mu_{2}, \quad \text { где } \mu(\xi)=\frac{L}{\xi} \operatorname{tg} \frac{\xi}{2} .
$$

Такой корень дает уравнение с параметром $\mu_{2}$, поскольку функция $\operatorname{tg}(\cdot)$ является возрастающей. Поскольку $\mu_{2}<0$, искомый минимальный корень $\xi_{1}$ локализован на интервале $(\pi, 2 \pi)$. Следовательно, значение минимального корня $\xi^{*}$ уравнения (3.17), локализованного на интервале $\left(0, \xi_{1}\right)$, удовлетворяет неравенству

$$
\xi^{*}<2 \pi
$$

Заметим, что величина $\xi$ в уравнениях траектории (3.9), (3.10) характеризует количество колебаний, которые произойдут в процессе движения на отрезке $\left[x_{0}, a\right]$, т. е. чем больше величина $\xi$, 
тем большее число колебаний будет иметь место. Тогда из неравенства (3.20) следует, что на оптимальной траектории, соответствующей минимальному корню $\xi^{*}$, пространственное колебательное движение наблюдателя длится меньше одного периода.

Таким образом, для окончательного решения задачи (2.3)-(2.7) необходимо найти корень уравнения (3.17) на интервале $\left(0, \xi_{1}\right)$. Для численного решения уравнения (3.17) авторами успешно применялся метод Ньютона. В качестве начального приближения выбиралась точка $\xi^{(0)}$, расположенная слева от точки $\xi_{1}: \xi^{(0)}=\xi_{1}-\delta \xi$. Малый положительный параметр $\delta \xi$ подбирался так, чтобы выполнялось неравенство $F_{2}\left(\xi^{(0)}\right)-F_{1}\left(\xi^{(0)}\right)>0$. Заметим, что при больших значениях весового коэффициента $\beta_{1}$ корень уравнения (3.17) расположен рядом с точкой $\xi_{1}$. Поэтому выбор в качестве начального приближения точки $\xi^{(0)}$ обеспечивает быструю сходимость метода Ньютона, что было подтверждено при численном моделировании.

\section{4. Условно-программный алгоритм управления наблюдателем}

Сформулируем алгоритм условно-программного управления наблюдателем, используя решение вспомогательной задачи оптимального управления, полученное для частного случая весовых коэффициентов в критерии (2.7). Будем считать, что обновление условно-программного управления происходит в моменты времени $t_{k}=\tau_{0}+k \Delta \tau, k=0,1, \ldots$, где $\Delta \tau$ - период обновления управления, кратный интервалу между наблюдениями. Пусть в момент $t_{k}$ имеются оценка фильтра $\widehat{R}\left(t_{k}\right)$ и ковариационная матрица ошибок оценивания фильтра $\gamma\left(t_{k}\right)$. Примем момент $t_{k}$ за начальный момент $t_{0}$ и решим вспомогательную задачу оптимального управления (2.3)-(2.7) при $\beta_{2}=0, \beta_{3}=0$ со следующими начальными условиями

$$
\begin{array}{ll}
x_{0}=\widehat{R}_{x}\left(t_{k}\right), & y_{0}=\widehat{R}_{y}\left(t_{k}\right), \\
z_{0}=\widehat{R}_{z}\left(t_{k}\right), & D\left(t_{0}\right)=\gamma^{-1}\left(t_{k}\right) .
\end{array}
$$

При решении вспомогательной задачи находится минимальный корень $\xi^{*}$ уравнения (3.17) и вычисляются значения параметров $\varepsilon\left(\xi^{*}\right), B_{1}\left(\xi^{*}\right)$ и $B_{2}\left(\xi^{*}\right)$, определяющих оптимальную траекторию (3.9), (3.10) с $x=x_{0}+V\left(t-t_{0}\right)$. Затем в течение времени $\Delta \tau$ наблюдатель движется по этой расчетной траектории, после чего происходит обновление программного управления путем решения задачи $(2.3)-(2.7)$ с $t_{0}=t_{k+1}$ и начальными условиями

$$
\begin{array}{ll}
x_{0}=\widehat{R}_{x}\left(t_{k+1}\right), & y_{0}=\widehat{R}_{y}\left(t_{k+1}\right), \\
z_{0}=\widehat{R}_{z}\left(t_{k+1}\right), & D\left(t_{0}\right)=\gamma^{-1}\left(t_{k+1}\right) .
\end{array}
$$

Управление наблюдателем заканчивается при $t=T$.

Таким образом, условно-программное управление наблюдателя, построенное в соответствии с описанным алгоритмом, представляет собой совокупность участков программного движения на временных отрезках $\left[t_{k}, t_{k+1}\right], k=0,1, \ldots$ На каждом из этих участков программное управление соответствует траектории (3.9), (3.10) с $x=\widehat{R}_{x}\left(t_{k}\right)+V\left(t-t_{k}\right)$ и параметрами $\varepsilon$, $B_{1}, B_{2}$, найденными из решения задачи (2.3)-(2.7) с начальными условиями (4.1). Реальная траектория наблюдателя при $t \in\left[t_{k}, t_{k+1}\right]$ описывается следующими уравнениями:

$$
\begin{aligned}
& R_{x}(t)=R_{x}\left(t_{k}\right)+V\left(t-t_{k}\right), \\
& R_{y}(t)=R_{y}\left(t_{k}\right)+y\left(\widehat{R}_{x}\left(t_{k}\right)+V\left(t-t_{k}\right)\right)-\widehat{R}_{y}\left(t_{k}\right), \\
& R_{z}(t)=R_{z}\left(t_{k}\right)+z\left(\widehat{R}_{x}\left(t_{k}\right)+V\left(t-t_{k}\right)\right)-\widehat{R}_{z}\left(t_{k}\right),
\end{aligned}
$$

где $y(\cdot), z(\cdot)$ - оптимальная программная траектория (3.9), (3.10).

Рассмотрим вопрос выбора интервала времени $\Delta \tau$ между обновлениями программного управления при численной реализации предложенного алгоритма управления наблюдателем. 
Заметим, что в рассматриваемой задаче численное решение вспомогательной задачи оптимального управления требует в несколько раз большего машинного времени, чем один такт работы алгоритма расширенного фильтра Калмана. По этой причине целесообразно решать задачу оптимального управления не в каждый такт времени (т. е. не после каждого измерения), а через несколько тактов работы алгоритма фильтрации. Целесообразность такого подхода оправдана еще и тем, что при малой длительности интервала между измерениями оценки вектора $R$, получаемые на двух последовательных тактах работы фильтра, мало отличаются друг от друга, и отвечающие этим оценкам оптимальные программные траектории наблюдателя будут практически совпадать. Исходя из этих соображений при проведении моделирования на ЭВМ, результаты которого описываются ниже, обновление программного управления производилось через 5 тактов работы алгоритма фильтрации, т. е. $\Delta \tau=5 \Delta$.

\section{5. Результаты численного моделирования}

Для проверки эффективности условно-программного управления наблюдателем, построенного на основании решения вспомогательной задачи оптимального управления (2.3)-(2.7), было проведено численное моделирование для частного случая $\beta_{2}=0, \beta_{3}=0$. В ходе моделирования исследовалось поведение расширенного фильтра Калмана при движении наблюдателя по траектории, которая строилась в соответствии с алгоритмом, описанным в предыдущем разделе.

Синтез условно-программного управления проводился для трех значений весового коэффициента $\beta_{1}$ при следующих начальных условиях

$$
\begin{array}{lll}
\tau_{0}=0 \mathrm{c}, & T=80 \mathrm{c}, & \Delta=0,2 \mathrm{c}, \\
\Delta \tau=1,0 \mathrm{c}, & V=100 \mathrm{~m} / \mathrm{c}, & \sigma_{0}=0,002 .
\end{array}
$$

Весовой коэффициент $\beta_{1}$ принимал значения $\beta_{1}=10^{-5} \mathrm{c}^{-1}, \beta_{1}=0,1 \mathrm{c}^{-1}$ и $\beta_{1}=10,0 \mathrm{c}^{-1}$.

Эффективность работы фильтра Калмана при движении по условно-программным траекториям оценивалась при помощи статистического моделирования, суть которого заключалась в следующем. Считалось, что компоненты вектора начального положения наблюдателя $R_{x}\left(\tau_{0}\right)$, $R_{y}\left(\tau_{0}\right), R_{z}\left(\tau_{0}\right)$ - независимые гауссовские случайные величины со средними $Z_{x}, Z_{y}, Z_{z}$ и среднеквадратическими отклонениями (с.к.о.) $S_{x}\left(\tau_{0}\right), S_{y}\left(\tau_{0}\right), S_{z}\left(\tau_{0}\right)$, численные значения которых определялись как

$$
\begin{array}{lll}
Z_{x}=-10000 \mathrm{M}, & Z_{y}=50 \mathrm{\textrm {M }}, & Z_{z}=-100 \mathrm{\textrm {M }} \\
S_{x}\left(\tau_{0}\right)=800 \mathrm{\textrm {M }}, & S_{y}\left(\tau_{0}\right)=20 \mathrm{\textrm {M }}, & S_{z}\left(\tau_{0}\right)=20 \mathrm{M}
\end{array}
$$

Предполагалось, что ковариационная матрица ошибок априорного оценивания $G$ имеет вид

$$
G=\left[\begin{array}{ccc}
S_{x}^{2}\left(\tau_{0}\right) & 0 & 0 \\
0 & S_{y}^{2}\left(\tau_{0}\right) & 0 \\
0 & 0 & S_{z}^{2}\left(\tau_{0}\right)
\end{array}\right] .
$$

В ходе моделирования условно-программное управление строилось для 5000 реализаций каждой из величин $R_{x}\left(\tau_{0}\right), R_{y}\left(\tau_{0}\right), R_{z}\left(\tau_{0}\right)$ и при движении по каждой реализовавшейся траектории в моменты обновления программного управления $t_{k}, k=0,1, \ldots$, вычислялись значения ошибки $e(t)$ оценивания $x$-компоненты вектора $R(t)$ :

$$
e\left(t_{k}\right)=\widehat{R}_{x}\left(t_{k}\right)-R_{x}\left(t_{k}\right) .
$$




\section{Результаты численного моделирования}

\begin{tabular}{|c|c|c|c|c|c|}
\hline & $\beta_{1}$ & $\bar{E}, \mathrm{~m}$ & $S_{x}(T), \mathrm{м}$ & промах $M(T), \mathrm{м}$ & расход $L(T), \mathrm{м}$ \\
\hline 1 & $10^{-6}$ & $-4,4$ & 142,5 & $0,95 \pm 0,67$ & $150,3 \pm 30,3$ \\
\hline 2 & 0,1 & 3,2 & 62,6 & $0,69 \pm 0,44$ & $179,4 \pm 26,6$ \\
\hline 3 & 10,0 & 0,97 & 21,1 & $0,59 \pm 0,34$ & $537,8 \pm 140,9$ \\
\hline
\end{tabular}

Кроме того, на каждой реализации вычислялись ошибка оценивания $E=e(T)$ в момент $T$ окончания процесса управления, значение расхода управляющего воздействия $L(T)$ и терминальный промах $M(T)$. Последние две величины находились по формулам

$$
L(t)=\int_{\tau_{0}}^{t}\left(\left|u_{1}(s)\right|+\left|u_{2}(s)\right|\right) d s, \quad M(T)=\sqrt{R_{y}^{2}(T)+R_{z}^{2}(T)} .
$$

Затем по набору реализаций определялась эволюция с.к.о. $S_{x}(t)$ ошибки оценивания компоненты $R_{x}(t)$, строился интегральный закон распределения случайной величины $E$, вычислялось ее среднее значение $\bar{E}$, а также среднее значение и с.к.о. величины $L(T)$.

Результаты статистического моделирования представлены в таблице выше и показаны на рис. 5-8. На всех рисунках график 1 построен для значения весового коэффициента $\beta_{1}=$ $10^{-6} \mathrm{c}^{-1}$, график $2-$ для $\beta_{1}=0,1 \mathrm{c}^{-1}$ и график $3-$ для $\beta_{1}=10,0 \mathrm{c}^{-1}$. На рис. 5 приведен вид интегрального закона распределения терминальной ошибки оценивания $E$, а на рис. 6 отражена эволюция с.к.о. ошибки оценивания $x$-компоненты вектора $R(t)$. Рисунок 7 демонстрирует распределения величины $L(T)$, которые позволяют судить о расходе управляющего воздействия к моменту $T$ при различных значениях коэффициента $\beta_{1}$.

На рис. 8 изображены типичные реализации условно-программных траекторий $R_{x}(t), R_{y}(t)$, $R_{z}(t)$ для трех значений весового коэффициента $\beta_{1}$. Выделена траектория наблюдателя, построенная для $\beta_{1}=10,0 \mathrm{c}^{-1}$, и показана ее проекция на плоскость OXZ.

Анализ результатов моделирования позволяет сделать следующие выводы.

1. Управление, построенное на основании решения вспомогательной задачи оптимального управления, при увеличении коэффициента $\beta_{1}$ оказывает существенное влияние на точность оценивания $x$-компоненты вектора $R$ с помощью расширенного фильтра Калмана. При этом выигрыш в точности оценивания величины $R_{x}(T)$ составляет один-два порядка по дисперсии.

2. Построенное управление при увеличении коэффициента $\beta_{1}$ уменьшает терминальный промах $M(T)$, обеспечивая более точное наведение.

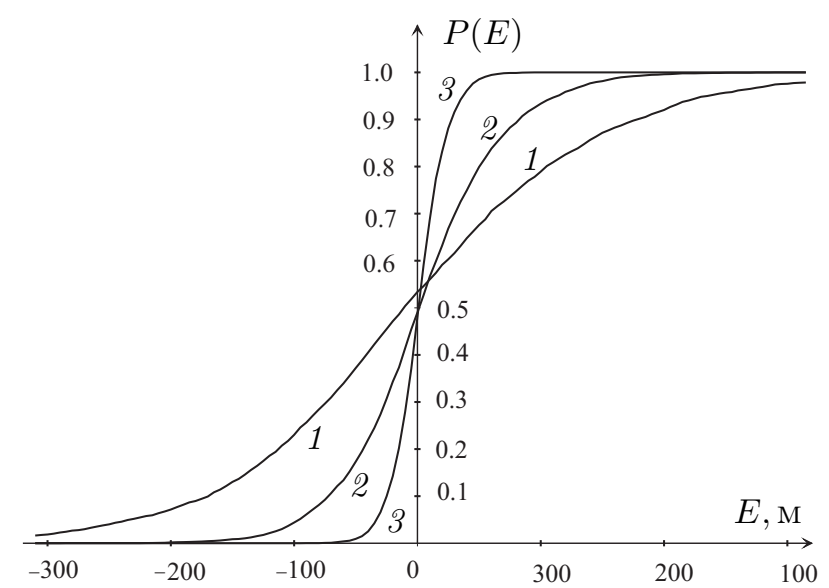

Рис. 5. Распределение терминальной ошибки оценивания $E$

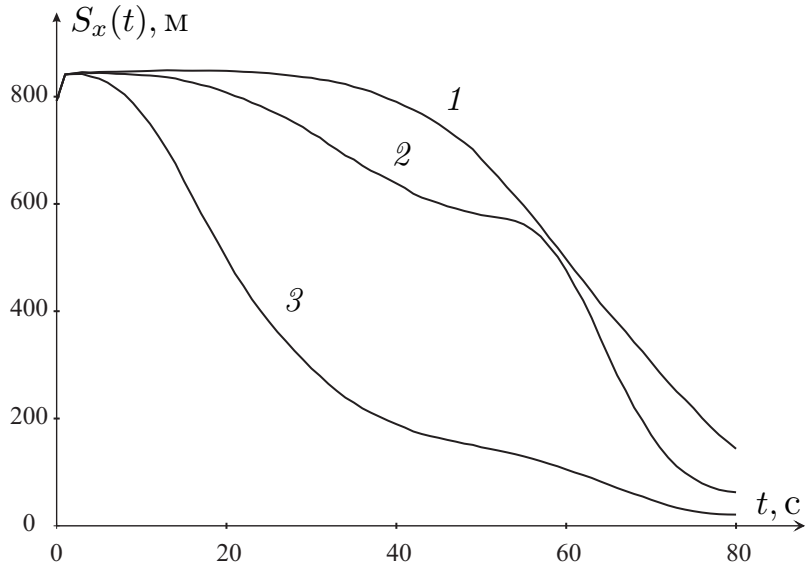

Рис. 6. Эволюция с.к.о. ошибки оценивания величины $R_{x}$ 


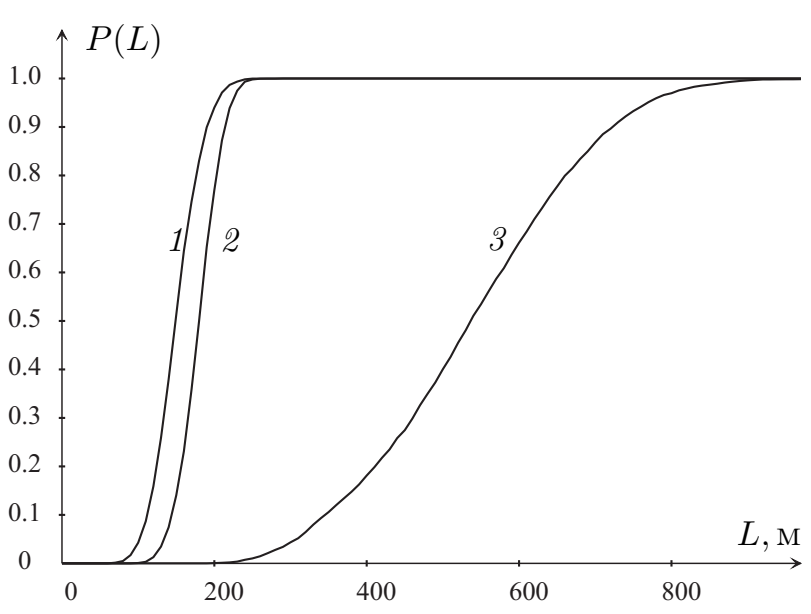

Рис. 7. Распределение величины $L(T)$

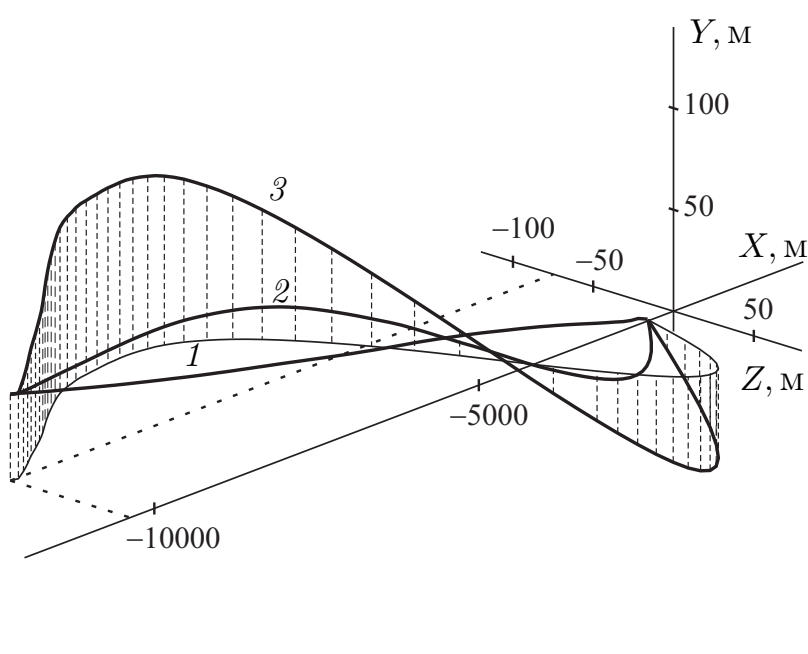

Рис. 8. Вид типичных реализаций условнопрограммных траекторий

3. Увеличение коэффициента $\beta_{1}$ приводит также к более быстрому убыванию с.к.о. $S_{x}(t)$ почти на всем отрезке управления. На отдельных реализациях это проявляется в ускорении сходимости оценки расширенного фильтра Калмана к точному значению.

4. Как видно из рис. 7 и таблицы, платой за повышение точности оценивания при увеличении коэффициента $\beta_{1}$ является рост расхода управляющего воздействия.

5. Для данных условий моделирования вид условно-программной траектории, функции $S_{x}(t)$ и распределения терминальной ошибки оценивания $E$ качественно меняется лишь при увеличении коэффициента $\beta_{1}$ в $10^{2}-10^{3}$ раз. Поэтому при построении управления, обеспечивающего как эффективную работу фильтра, так и приемлемый расход управления, возможный диапазон значений $\beta_{1}$ достаточно широк и нет необходимости в точном подборе этого коэффициента при настройке алгоритма условно-программного управления.

\section{Заключение}

1. Рассмотрена пространственная задача управления наблюдателем, который оценивает вектор своих координат по дискретным угломерным наблюдениям при помощи расширенного фильтра Калмана.

2. Решение задачи осуществлялось на классе условно-программных управлений, что позволило свести задачу синтеза траектории наблюдателя к решению вспомогательной задачи оптимального управления с критерием, обеспечивающим эффективную работу фильтра, и начальными условиями, обновляющимися после каждого такта (или после нескольких тактов) работы фильтра.

3. Получено аналитическое решение вспомогательной задачи оптимального управления, найден вид оптимальной траектории наблюдателя, проведено исследование оптимального решения для частного случая задачи.

4. Сформулирован условно-программный алгоритм управления наблюдателем.

5. Эффективность построенного управления наблюдателем проверена при помощи статистического моделирования. Анализ полученных результатов показал, что применение траекторного управления наблюдениями в рассматриваемой задаче повышает точность оценивания координат на один-два порядка по дисперсии и ускоряет сходимость оценок расширенного фильтра Калмана. 


\section{СПИСОК ЛИТЕРАТУРЫ}

1. Nardone S.C., Aidala V.J. Optimization of observer trajectories for bearings-only target localization // IEEE Trans. Aerospace and Electronic Syst. 1981. Vol. AES-17, no. 2. P. 162-166. doi: 10.1109/TAES.1981.309141.

2. Liu P.T. An optimum approach in target tracking with bearing measurements // J. Optim. Theory Appl. 1988. Vol. 56, no. 2. P. 205-214. doi: 10.1007/BF00939407.

3. Oshman Y., Davidson V. Optimization of observer trajectories for bearings-only target localization // IEEE Trans. Aerospace and Electronic Syst. 1999. Vol. 35, no. 3. P. 892-902. doi: 10.1109/7.784059 .

4. Miller A., Miller B. Tracking the UAV trajectory on the basis of bearing-only observations // Proc. 53rd IEEE Conf. Decision and Control - CDC2014. 2014. N Y: IEEE, 2014. P. 4178-4184. doi: 10.1109/CDC.2014.7040040.

5. Zajic T., Mahler R.P.S. Particle-systems implementation of the PHD multitarget tracking filter // Proc. SPIE Conf. Series. 2003. Vol. 5096. P. 291-299.

6. Lanneuville D., Houssineau J. Passive multi-target tracking with GM-PHD filter // Proc. 13th Conf. Inform. Fusion. N Y: IEEE, 2010. P. 1-7. doi: 10.1109/ICIF.2010.5711954.

7. Bar-Shalom Y., Willett P.K., Tian X. Tracking and data fusion: A handbook of algorithms. Storrs, CT: YBS-Press, 2011. 1235 p.

8. Lin X., Kirubarajan T., Bar-Shalom Y. [et al.] Comparison of EKF pseudomeasurement and particle filters for a bearing-only target tracking problem // Proc. SPIE Conf. Series Signal and Data Processing of Small Targets / eds. Oliver E. Drummond. 2002. Vol. 4728. P. 240-250. doi: $10.1117 / 12.478508$.

9. Андреев K.В., Рубинович Е.Я. Траекторное управление наблюдателем за мобильной целью по угломерной информации // Автоматика и телемеаника. 2016. № 1. С. 134-162.

10. Miller S.A., Harris Z.A., Chong E.K.P. A POMDP framework for coordinated guidance of autonomous uavs for multitarget tracking // EURASIP J. Adv. Signal Process. Special issue on signal processing advances in robots and autonomy. 2009. Article ID: 724597. 17 p. doi: 10.1155/2009/724597.

11. Емельянов Об одной задаче траекторного управления наблюдениями // Автоматика и телемеханика. 1997. Вып. 10. С. 47-58.

12. Liptser R.S., Shiryaev A.N. Statistics of random processes. NY: Springer-Verlag, 1978. 457 p. doi: $10.1007 / 978-3-662-13043-8$.

Поступила 15.01.2020

После доработки 2.02.2020

Принята к публикации 10.02.2020

Емельянов Дмитрий Дмитриевич

канд. техн. наук

ведущий науч. сотрудник

STFC Rutherford Appleton Laboratory

Harwell, OX11 0QX UK

e-mail: d.emeliyanov@outlook.com

Рубинович Евгений Яковлевич

Д-р техн. наук, профессор

главный науч. сотрудник

Институт проблем управления им. В. А. Трапезникова РАН

г. Москва

e-mail: rubinvch@ipu.rssi.ru

\section{REFERENCES}

1. Nardone S.C., Aidala V.J. Optimization of observer trajectories for bearings-only target localization. IEEE Trans. Aerospace and Electronic Syst., 1981, vol. AES-17, no. 2, pp. 162-166. doi: 10.1109/TAES.1981.309141.

2. Liu P.T. An optimum approach in target tracking with bearing measurements. J. Optim. Theory Appl., 1988, vol. 56, no. 2, pp. 205-214. doi: 10.1007/BF00939407. 
3. Oshman Y., Davidson V. Optimization of observer trajectories for bearings-only target localization. IEEE Trans. Aerospace and Electronic Syst., 1999, vol. 35, no. 3, pp. 892-902. doi: 10.1109/7.784059.

4. Miller A., Miller B. Tracking the UAV trajectory on the basis of bearing-only obervations. In: Proc. 53rd IEEE Conf. Decision and Control - CDC2014, N Y: IEEE, 2014. pp. 4178-4184. doi: 10.1109/CDC.2014.7040040 .

5. Zajic T., Mahler R.P.S. Particle-systems implementation of the PHD multitarget tracking filter. In: Proc. SPIE Conf. Series, 2003, vol. 5096, pp. 291-299.

6. Lanneuville D., Houssineau J. Passive multi-target tracking with GM-PHD filter. In: Proc. 13th Conf. Inform. Fusion, N Y: IEEE, 2010, pp. 1-7. doi: 10.1109/ICIF.2010.5711954.

7. Bar-Shalom Y., Willett P.K., Tian X. Tracking and data fusion: A handbook of algorithms. Storrs, CT: YBS-Press, 2011, 1235 p. ISBN: 0964831279.

8. Lin X., Kirubarajan T., Bar-Shalom Y., et al. Comparison of EKF pseudomeasurement and particle filters for a bearing-only target tracking problem. In: Proc. SPIE Conf. Series Signal and Data Processing of Small Targets / ed. Oliver E. Drummond, 2002, vol. 4728, pp. 240-250. doi: 10.1117/12.478508.

9. Andreev K.V., Rubinovich E.Ya. Moving Observer trajectory control by angular measurements in tracking problem. Autom. Remote Control, 2016, vol. 77, no. 1, pp. 106-129. doi: 10.1134/S0005117916010069.

10. Miller S.A., Harris Z.A., Chong E.K.P. A POMDP framework for coordinated guidance of autonomous UAVs for multitarget tracking. EURASIP J. Adv. Signal Process., 2009, Article ID: 724597, 17 p. doi: $10.1155 / 2009 / 724597$.

11. Emel'yanov D.D. To the problem of trajectory control of observations. Autom. Remote Control, 1997, vol. 58, no. 10, pp. 1592-1600.

12. Liptser R.S., Shiryaev A.N. Statistics of random processes. Berlin: Springer, 1978. 457 p. doi: 10.1007/978-3-662-13043-8 .

Received January 15, 2020

Revised February 2, 2020

Accepted February 10, 2020

Funding Agency: This work was partially supported by the Program fundamental research No. 7 of the Presidium of the Russian Academy of Sciences.

Dmitry Dmitrievich Emeliyanov, Cand. Sci. (Engineering), Particle Physics Department of STFC Rutherford Appleton Laboratory, Harwell Science and Innovation Campus, OX11 0QX UK, e-mail: d.emeliyanov@outlook.com.

Evgeny Yakovlevich Rubinovich, Dr. Sci. Engineering, Prof., Trapeznikov Institute of Control Sciences of the Russian Academy of Sciences, Moscow, 117997 Russia, e-mail: rubinvch@ipu.rssi.ru .

Cite this article as: D. D. Emeliyanov, E. Ya. Rubinovich. Trajectory control of 3D observations from an UAV by angular measurements, Trudy Instituta Matematiki i Mekhaniki URO RAN, 2020, vol. 26, no. 1 , pp. $112-130$. 